\title{
Effects of Solvent and Solute Polarizability on the Reorganization Energy of Electron Transfer
}

\author{
Shikha Gupta and Dmitry V. Matyushov* \\ Department of Chemistry and Biochemistry and the Center for the Study of Early Events in Photosynthesis, \\ Arizona State University, PO Box 871604, Tempe, Arizona 85287-1604
}

Received: August 11, 2003; In Final Form: December 10, 2003

\begin{abstract}
We report Monte Carlo simulations of the effect of solute and solvent polarizability on the solvent reorganization energy of intramolecular electron transfer. In the first set of simulations, the polarizability of the solvent is varied at constant permanent dipole of the solvent molecules (high-frequency dielectric constants is in the range 1-2.5). The reorganization energy is calculated on the solvent configurations around a nonpolar solute (charge separation transition) and around a dipolar solute (charge recombination transition). In both cases, the variation of the solvent reorganization energy does not exceed 30\%, a change much smaller than predicted by dielectric continuum models. In the second set of simulations, the solute polarizability in the chargeseparated state was varied while keeping the initial state for charge separation at zero dipole moment and polarizability. The gap between the charge-separation and charge recombination reorganization energies widens substantially with increasing difference in the polarizability of the initial and final charge-transfer states. Both the effect of solute and solvent polarizability can be accurately described by analytical theories of solvent reorganization.
\end{abstract}

\section{Introduction}

Existing theories of electron transfer (ET) reactions predict a substantial effect of the solvent polarizability on the reaction activation barrier. The main portion of this effect arises from the solvent reorganization energy $\lambda_{\mathrm{s}}$. The classical Marcus formulation $^{1}$ predicts that for widely separated donor and acceptor units (intermolecular ET) $\lambda_{\mathrm{s}}$ is proportional to the Pekar factor

$$
c_{0}=\epsilon_{\infty}^{-1}-\epsilon_{\mathrm{s}}^{-1}
$$

where $\epsilon_{\infty}$ and $\epsilon_{\mathrm{s}}$ are the high-frequency and static dielectric constants, respectively. In highly polar solvents with $\epsilon_{\mathrm{s}} \gg 1$ the factor $1 / \epsilon_{\mathrm{s}}$ in $c_{0}$ is insignificant, and the reorganization energy depends on the solvent predominantly through $\epsilon_{\infty}$. The magnitude of $\lambda_{\mathrm{s}}$ then drops by about a factor of 2 in going from $\epsilon_{\infty}=$ 1 to $\epsilon_{\infty}=2$ common for molecular solvents. When the distance between the donor and acceptor is small compared to the characteristic radius of the donor-acceptor complex (DAC) (intramolecular ET), the relevant solvent polarity factor is defined by the Onsager reaction field ${ }^{2}$ (Lippert-Mataga equa$\operatorname{tion}^{3}$ )

$$
f=\frac{\epsilon_{\mathrm{s}}-1}{2 \epsilon_{\mathrm{s}}+1}-\frac{\epsilon_{\infty}-1}{2 \epsilon_{\infty}+1}
$$

In this case, the reorganization energy drops by a factor of 1.7 when the high-frequency dielectric constant changes from $\epsilon_{\infty}$ $=1$ to $\epsilon_{\infty}=2$ at $\epsilon_{\mathrm{s}} \gg 1$. Most real ET configurations fall between these two limiting cases, and according to dielectric solvation theories, one should expect a significant dependence of the activation barrier on $\epsilon_{\infty}$.

* To whom correspondence should be addressed. E-mail: dmitrym@ asu.edu.
Despite its significance for the formulation of theories of solvent effect on ET activation, the dependence of $\lambda_{\mathrm{s}}$ on $\epsilon_{\infty}$ has never been tested experimentally. The common experimental setup involves changing solvent for the same ET system, which alters both $\epsilon_{\infty}$ and $\epsilon_{\mathrm{S}}$ along with other solvent properties. Linear trends of the reorganization energy with either $c_{0}^{4}$ or $f^{5-7}$ are often observed and are interpreted to support the predictions of dielectric theories. However, a strong dependence of $\lambda_{\mathrm{s}}$ on $\epsilon_{\infty}$ substantially affects the calculated values of the entropy and volume of reorganization for which dielectric models do not perform well. ${ }^{8,9}$ This fact brings up the question of the actual dependence of $\lambda_{\mathrm{s}}$ on the high-frequency dielectric constant.

The high-frequency dielectric constant $\epsilon_{\infty}$ is related to the solvent molecular polarizability through the Clausius-Mossotti relation. ${ }^{10}$ Changes in molecular polarizability $\alpha$ is the main source of the variation of $\epsilon_{\infty}$ observed among molecular solvents at ambient conditions. The problem of the effect of solvent polarizability, and, related to it, the problem of the effect of $\epsilon_{\infty}$ on solvation thermodynamics, is also relevant to theoretical modeling of reorganization parameters of ET. The inclusion of solvent polarizability in condensed-phase calculations and/or simulations is often hard because of the many-body character of polarizability effects. An approach often employed in theoretical algorithms to go around the problem is to perform calculations at zero solvent polarizability and then rescale the results according to the predictions of dielectric models. An alternative approach, widely used in calculations involving complex molecular geometries, ${ }^{11,12}$ is to represent the reorganization energy as the difference of two equilibrium solvation free energies calculated for solvation in dielectric media characterized by the dielectric constants $\epsilon_{\infty}$ and $\epsilon_{\mathrm{s}}$.

In the absence of direct experimental evidence on the effect of solvent polarizability on ET rates, computer simulations may provide the necessary insight. The first microscopic study of the polarizability effect was given in the simulations by King 
and Warshel, ${ }^{13}$ who concluded that increasing solvent polarizability markedly reduces $\lambda_{\mathrm{s}}$. This conclusion was challenged by Berne and co-workers ${ }^{14,15}$ who carried out full self-consistent simulations of electronic transitions in formaldehyde in two types of water models, polarizable and nonpolarizable. Their conclusion was that the change from a polarizable to a nonpolarizable water model does not strongly affect $\lambda_{\mathrm{s}}$. These results were further supported in simulations by Ando, who compared a flexible-charge model of water (TIP3P-FQ) with its fixed-charge analogue (TIP3P) for the calculation of $\lambda_{\mathrm{s}}{ }^{16}$ The polarizable and nonpolarizable models of water used in these simulations were parametrized to give the observable average dipole moment of bulk water $(\simeq 2.5 \mathrm{D})$, i.e., nonpolarizable molecules had a higher permanent dipole moment than molecules in polarizable solvents.

The conclusion from both Berne et al. ${ }^{14,15}$ and Ando ${ }^{16}$ simulations is that, for the purpose of calculating the reorganization energy, the full polarizable solvent is equivalent to a nonpolarizable solvent with an effective permanent dipole moment. This result is not that surprising in view of welldeveloped self-consistent models of polarizable solvents ${ }^{17}$ employing the idea that a nonpolarizable solvents with an effective permanent dipole is equivalent from the perspective of thermodynamic properties to a polarizable solvent. The question we address in this study goes one step further: What is the effect of the actual change of $\epsilon_{\infty}$ at constant permanent dipole of the solvent on $\lambda_{\mathrm{s}}$ ? We report Monte Carlo (MC) simulations of dipolar solvation in solvents with varying polarizability. The simulation results indicate that the reorganization energy in strongly polar solvents does decay with increasing $\epsilon_{\infty}$. However, the change of $\lambda_{\mathrm{s}}$ when $\epsilon_{\infty}$ changes from 1 to 2.5 does not exceed $30 \%$, a much weaker variation than one would expect from the $f$ factor in eq 2 .

A general understanding of the effect of molecular polarizability on the kinetics of ET reactions cannot be achieved without addressing the problem of the effect of solute polarizability on the ET activation barrier. This part of the problem has a new flavor due to the fact that electronic transitions can actually induce quite substantial changes in the molecular polarizability of the DAC. ${ }^{18}$ Analytical theories ${ }^{19,20}$ predict a substantial effect of the polarizability change on the activation barrier. The key origin of this effect is the change in the solvent reorganization energy with the solute polarizability. The second part of this study reports direct MC simulations of the solvent reorganization energy for charge separation (CS) and charge recombination $(\mathrm{CR})$ transitions accompanied by the alteration of both the solute dipole moment and dipolar polarizability. A good agreement between analytical theory and computer simulations is achieved.

The rest of the paper is organized as follows. A general discussion of ET thermodynamics in section 2 is followed in section 3 by a more specific formulation for intramolecular transitions in dipolar DACs. Section 4 presents the results of MC simulations in which the solvent polarizability and the polarizability of the final ET state are separately varied. We conclude with the discussion of results in section 5 .

\section{Problem Formulation}

The electronic states of the donor and acceptor in a DAC are coupled to the electrostatic potential of the solvent produced by the electronic and nuclear charges on the solvent molecules. The electronic shells of both the solvent and the solute are inherently polarizable resulting in the creation of induced charges equilibrated to the instantaneous electric field. The probability of realizing a particular equilibrium or nonequilibrium configuration of nuclear charges and the equilibrium distribution of induced charges is determined by the instantaneous (partial) free energy of a condensed-phase system. This free energy, obtained by tracing out the electronic degrees of freedom, ${ }^{21,22}$ depends on the instantaneous nuclear configuration of the system. The free energy consists of the energy of all intermolecular interactions and the free energy of polarization of the electronic shells by the internal electric field in the system.

For problems related to electronic transitions in molecules dissolved in condensed-phase solvents, one considers the instantaneous free energy of a solute in its electronic state $i$ :

$$
E_{i}[\mathbf{Q}]=I_{i}+V_{i}(\mathbf{Q})+\Pi_{i}(\mathbf{Q})
$$

Here $\mathbf{Q}$ denotes the manifold of all nuclear coordinates in the system, $I_{i}$ is the vacuum energy of the solute in its $i$ th electronic state, and the last two terms describe the solute-solvent interaction and the free energy of solute polarization, respectively. In molecular solvents, the manifold of nuclear configurations is defined by molecular coordinates $\mathbf{q}$ and molecular orientations $\omega: \mathbf{Q}=\{\mathbf{q}, \boldsymbol{\omega}\}$.

The energy of interaction $V_{i}$ between a solute and a polar molecular solvent consists of the coupling of the solute electric field $\mathbf{E}_{0 i}$ to the solvent nuclear polarization $\mathbf{P}_{\mathrm{n}}$ (first summand in eq 4) and nonpolar interactions (dispersion and induction forces, second summand in eq 4)

$$
V_{i}(\mathbf{Q})=-\int \mathbf{E}_{0 i}(\mathbf{r}) \cdot \mathbf{P}_{\mathrm{n}}(\mathbf{r}) \mathrm{d} \mathbf{r}+V_{i}^{\mathrm{np}}(\mathbf{q})
$$

Thermal fluctuations of $V_{i}(\mathbf{Q})$ result in radiationless transitions (ET reactions) or bring about inhomogeneous broadening of optical lines. The traditional Marcus - Hush formulation ${ }^{1}$ focuses on the first term in eq 4 as the main source of both effects. Only the first two cumulants in $V_{i}(\mathbf{Q})$ define the rate constant or optical band-shape ${ }^{23}$ in this formulation (Gaussian approximation). Correspondingly, the theory is formulated in terms of the average vertical transition energy $\Delta F_{i}(i=1$ for CS and $i=2$ for $\mathrm{CR}$ ) and the variance $\sigma(\Delta V)$ of the distribution of energy gaps $\Delta V(\mathbf{Q})=V_{2}(\mathbf{Q})-V_{1}(\mathbf{Q})$. The latter property defines the solvent reorganization energy:

$$
\lambda_{\mathrm{s}}=\beta \sigma(\Delta V)^{2} / 2
$$

where $\beta=1 / k_{\mathrm{B}} T, k_{\mathrm{B}}$ is Boltzmann's constant, and $T$ is the temperature. The free energy barrier for ET is given in terms of two parameters of the Gaussian approximation as

$$
\Delta F_{i}^{\mathrm{act}}=\Delta F_{i}^{2} / 4 \lambda_{\mathrm{s}}
$$

The average vertical gap is usually split into the equilibrium free energy gap $\Delta F_{0}$ and the reorganization energy: $\Delta F=\Delta F_{0}$ $\pm \lambda_{\mathrm{s}}$; "+" and "-" correspond to $i=1(\mathrm{CS})$ and $i=2(\mathrm{CR})$, respectively.

The Gaussian fluctuations of the solute-solvent coupling $V_{i}(\mathbf{Q})$ in eq 4 is the result of thermal fluctuations of the solvent nuclear polarization (polar coupling) and of the nonpolar interaction potential (nonpolar coupling). The former are mainly generated by correlated orientational motions of the solvent permanent dipoles, whereas the latter are essentially due to the density fluctuations of the solvent in solute's vicinity. If these two stochastic processes are uncoupled, one can represent the reorganization energy as a sum of polar $\left(\lambda_{\mathrm{p}}\right)$ and nonpolar $\left(\lambda_{\mathrm{np}}\right)$ contributions ${ }^{24}$ 


$$
\lambda_{\mathrm{s}}=\lambda_{\mathrm{p}}+\lambda_{\mathrm{np}}
$$

In the Marcus - Hush formulation, $\lambda_{\mathrm{np}}=0$ and $\lambda_{\mathrm{s}}=\lambda_{\mathrm{p}}$.

A formula for $\lambda_{\mathrm{p}}$, which is the main component of $\lambda_{\mathrm{s}}$ for ET in polar solvents, can be written in terms of the polarization correlation function

$$
\lambda_{\mathrm{p}}=(\beta / 2) \sum_{\alpha, \beta} \int \Delta E_{0 \alpha}\left(\mathbf{r}^{\prime}\right) \Delta E_{0 \beta}\left(\mathbf{r}^{\prime \prime}\right)\left\langle\delta P_{n \alpha}\left(\mathbf{r}^{\prime}\right) \delta P_{n \beta}\left(\mathbf{r}^{\prime \prime}\right)\right\rangle \mathrm{d} \mathbf{r}^{\prime} \mathrm{d} \mathbf{r}^{\prime \prime}
$$

where $\alpha, \beta$ subscripts stand for Cartesian components, angular brackets mean ensemble average, and $\Delta \mathbf{E}_{0}=\mathbf{E}_{02}-\mathbf{E}_{01}$. The response is local in the continuum approximation and is proportional to the response function of the nuclear polarization $\chi_{\mathrm{p}}$ :

$$
\left\langle\delta P_{n \alpha}\left(\mathbf{r}^{\prime}\right) \delta P_{n \beta}\left(\mathbf{r}^{\prime \prime}\right)\right\rangle=\chi_{\mathrm{p}} \delta_{\alpha, \beta} \delta\left(\mathbf{r}^{\prime}-\mathbf{r}^{\prime \prime}\right)
$$

When the correlation length of the molecular response is comparable to the characteristic distance of decay of the difference field $\Delta \mathbf{E}_{0}$, the response function should be taken in a nonlocal form, $\chi_{\mathrm{p}}\left(\mathbf{r}^{\prime}-\mathbf{r}^{\prime \prime}\right)$. In either case, it is generally difficult to extract the response function of the nuclear polarization only, and some approximations are commonly adopted. The most widely used approach is to obtain $\chi_{\mathrm{p}}$ by subtracting the response function of the electronic solvent polarization, $\chi_{\mathrm{e}}$, from the total response function $\chi$ incorporating both the nuclear and electronic polarization

$$
\chi_{\mathrm{p}}=\chi-\chi_{\mathrm{e}}
$$

For longitudinal fields $\Delta \mathbf{E}_{0}$ this approximation results in the appearance of the Pekar factor in $\lambda_{\mathrm{p}}$ in eq 1 whereas the dipolar field $\Delta \mathbf{E}_{0}$ gives the $f$ factor in eq 2 .

The Marcus-Hush formulation (eqs 4-6) applies only if the polarization energy $\Pi_{i}(\mathbf{Q})$ in eq 3 is either small or does not change with the electronic transition. The polarization term is quadratic in the solvent nuclear polarization. The donoracceptor energy gap $\Delta E[\mathbf{Q}]=E_{2}(\mathbf{Q})-E_{1}(\mathbf{Q})$ is then a bilinear function of the nuclear polarization. A formal description of this situation leads to a three-parameter theory of ET (Q model) $)^{25}$ with nonparabolic free energy surfaces and the activation barrier given in the form

$$
\Delta F_{i}^{\mathrm{act}}=\left|\alpha_{i}\right|\left(\sqrt{\left|\Delta F_{0}-\lambda_{1} \alpha_{1}^{2} / \alpha_{2}\right|}-\sqrt{\left|\alpha_{i}\right| \lambda_{i}}\right)^{2}
$$

The main distinction of the new description compared to the Marcus-Hush formalism is the appearance of two different reorganization energies $\lambda_{i}$ for the forward and backward ET transitions. As above, $\Delta F_{0}$ is the equilibrium free energy gap. The parameters $\alpha_{i}$ control the extent of deviation between the two reorganization energies. Both reorganization energies are defined through the variance of $\Delta E$ evaluated at the equilibrium distribution of the solvent in each state

$$
\lambda_{i}=\beta \sigma_{i}(\Delta E)^{2} / 2
$$

where

$$
\Delta E=\Delta I+\Delta V+\Delta \Pi
$$

The parameter $\alpha_{1}$ is defined by the relation

$$
\alpha_{1}=\left(\sqrt[3]{\lambda_{1} / \lambda_{2}}-1\right)^{-1}
$$

and $\alpha_{2}$ is defined by the relation $\alpha_{2}=1+\alpha_{1}$. At $\lambda_{1} \simeq \lambda_{2}$ one obtains $\alpha_{1} \rightarrow \infty$ and eq 11 transforms into eq 6 . The main distinction between eqs 5 and 12 is that in the latter the variance is taken on the difference energy $\Delta E$ including the change in the polarization energy $\Pi$.

\section{Dipole Solvation}

The instantaneous free energies $E_{i}[\mathbf{Q}]$ are defined in terms of a partial trace $\left(\operatorname{Tr}_{\mathrm{el}}\right)$ of the density matrix in the $i$ th electronic state of the solute over the electronic degrees of freedom of the solvent molecules

$$
\mathrm{e}^{-\beta E_{i}[\mathbf{Q}]}=\operatorname{Tr}_{\mathrm{el}}\left(\mathrm{e}^{-\beta H_{i}}\right)
$$

where $H_{i}$ is the Hamiltonian of the solute in the $i$ th state and the solvent. The necessity to generate free energies of the initial and final ET states at each nonequilibrium configuration of the nuclei substantially complicates the treatment of the problem by both computer simulations and analytical theories. Equation 15 can be solved analytically ${ }^{21}$ for a few model systems, e.g., for the solvent of Drude oscillators modeling the induced solvent dipoles. ${ }^{26-28}$ We start our development with the result of analytical integration in eq 15 . The variance of analytically obtained energies $E_{i}[\mathbf{Q}]$ is calculated on the nuclear configurations generated by Metropolis MC simulations ${ }^{29}$ used to calculate the reorganization energies. This two-step procedure allows us to avoid the complex problem of calculating the instantaneous free energies from simulations on one hand and to obtain the average over the many-body nuclear configurations, hard for analytical treatments, from computer simulations better suited for this purpose on the other.

Treatments of polarizable systems are still very demanding computationally. ${ }^{30}$ To obtain good statistics for the second cumulant of the solute-solvent interaction potential, we consider here a classical model system of a polarizable point dipole centered in a spherical solute in a solvent of polarizable dipolar hard spheres. ${ }^{2,3,31-33}$ For this model, the integration over the Drude amplitudes in eq 15 leads to the following exact expression for the instantaneous solute-solvent coupling ${ }^{19,21}$

$$
V_{i}\left[\mathbf{R}_{\mathrm{p}}, \mathbf{q}\right]=-\mathbf{m}_{0 i} \cdot \mathbf{f}_{\mathrm{ei}}(\mathbf{q}) \cdot \mathbf{R}_{\mathrm{p}}-\frac{1}{2} \mathbf{m}_{0 i} \cdot \mathbf{R}_{\infty}(\mathbf{q}) \cdot \mathbf{f}_{\mathrm{ei}}(\mathbf{q}) \cdot \mathbf{m}_{0 i}
$$

In eq $16, \mathbf{R}_{\infty}(\mathbf{q})$ is the reaction field of the induced solvent dipoles depending on the instantaneous configuration of the molecular coordinates $\mathbf{q}$ :

$$
\mathbf{R}_{\infty}(\mathbf{q})=\sum_{j, k} \mathbf{T}_{0 j} \cdot \alpha(\mathbf{1}-\alpha \mathbf{T})_{j k}{ }^{-1} \cdot \mathbf{T}_{k 0}
$$

The tensor $\mathbf{T}_{j k}=\nabla_{j} \nabla_{k}\left|\mathbf{r}_{j}-\mathbf{r}_{k}\right|^{-1}$ is the dipolar tensor between the $j$ th and $k$ th solvent molecules; $\mathbf{T}_{0 j}$ is the solute-solvent dipolar tensor. The matrix $\alpha(\mathbf{1}-\alpha \mathbf{T})^{-1}$ appears as a result of many-particle induction effects which, in the self-consistent approximation, ${ }^{17}$ renormalize the vacuum polarizability $\alpha$ to its condensed-phase value

$$
\alpha^{\prime}=\left\langle\alpha(\mathbf{1}-\alpha \mathbf{T})^{-1}\right\rangle
$$

The induction effects are also responsible for the enhancement of the solute-solvent coupling through the 2-rank tensor

$$
\mathbf{f}_{\mathrm{ei}}(\mathbf{q})=\left[\mathbf{1}-2 \alpha_{0 i} \mathbf{R}_{\infty}(\mathbf{q})\right]^{-1}
$$

Finally, the solute-solvent coupling $V_{i}\left[\mathbf{R}_{\mathrm{p}}, \mathbf{q}\right]$ depends on molecular orientations through the reaction field of the nuclear 
solvent polarization $\mathbf{R}_{\mathrm{p}}$ defined as the combined electric field of the solvent condensed-phase dipoles $\mathbf{m}_{j}^{\prime}$

$$
\mathbf{R}_{\mathrm{p}}=\sum_{j} \mathbf{T}_{0 j} \cdot \mathbf{m}_{j}^{\prime}
$$

The dipole moment $\mathbf{m}^{\prime}$ is usually defined in liquid-state theories within a self-consistent algorithm. ${ }^{17}$ In simulations, $\mathbf{m}^{\prime}$ is given by the sum of the permanent dipole $\mathbf{m}$ and the induced dipole created by the field of all other dipoles in the solvent

$$
\mathbf{m}_{j}^{\prime}=\mathbf{m}_{j}+\sum_{k l} \alpha(\mathbf{1}-\alpha \mathbf{T})_{j k}{ }^{-1} \cdot \mathbf{T}_{k l} \cdot \mathbf{m}_{l}
$$

In analyzing the simulation results, the term $\alpha(\mathbf{1}-\alpha \mathbf{T})^{-1}$ in eq 21 was replaced by the self-consistent polarizability $\alpha^{\prime}$ according to eq 18 .

The integration over the solvent and solute induced dipoles in eq 15 also produces the free energy of polarization of the solute by the nuclear field $\mathbf{R}_{\mathrm{p}}$ of the solvent

$$
\Pi_{i}\left[\mathbf{R}_{\mathrm{p}}\right]=-\frac{\alpha_{0 i}}{2} \mathbf{R}_{\mathrm{p}} \cdot \mathbf{f}_{\mathrm{ei}} \cdot \mathbf{R}_{\mathrm{p}}
$$

The dependence of this term on the state of the solute through the solute dipolar polarizability $\alpha_{0 i}$ necessitates the transition from the Marcus-Hush formulation in eq 6 to the threeparameter description of the Q model in eq 11 .

The second term in eq 16 represents the solute-solvent induction coupling. If the fluctuations of the reaction field $\mathbf{R}_{\mathrm{p}}$ are statistically independent from the fluctuations of the reaction field $\mathbf{R}_{\infty}$, the solvent reorganization energy splits into dipolar and induction components

$$
\lambda_{i}=\lambda_{\mathrm{p}, i}+\lambda_{\text {ind }}
$$

The reorganization energy components are related to the variance of $\Delta V_{\mathrm{p}}$ (first summand in eq 16) and $\Delta V_{\text {ind }}$ (second summand in eq 16) according to eq 12. Equation 23 is a specific case of eq 7 when nonpolar interactions include only the induction forces.

If the nuclear reaction field $\mathbf{R}_{\mathrm{p}}$ obeys the Gaussian statistics, the variance of the potential difference $\Delta V_{\mathrm{p}}$ can be calculated exactly: ${ }^{19,25}$

$$
\lambda_{\mathrm{p}, i}=a_{\mathrm{p}}\left(f_{i} / f_{\mathrm{ei}}\right)\left[\Delta \tilde{\mathbf{m}}_{0}+2 a_{\mathrm{p}} f_{i} \Delta \tilde{\alpha}_{0} \mathbf{m}_{0 i}\right]^{2}
$$

The strength of dipole solvation is determined by solute's dipole moment and the response coefficient $a$ such that the chemical potential of solvation is $\mu=-a m_{0}^{2}$. The electronic and nuclear contributions to $\mu$ have the corresponding response coefficients $a_{\mathrm{e}}$ and $a_{\mathrm{p}}$. The chemical potential of electronic, $\mu_{\mathrm{e}}$, and nuclear, $\mu_{\mathrm{p}}$, solvation are then $\mu_{\mathrm{e}, \mathrm{p}}=-a_{\mathrm{e}, \mathrm{p}} m_{0}^{2}$. Also the parameters $f_{\mathrm{e}}$ and $f_{i}$ are given in terms of these response coefficients and the solute polarizability according to the following relations

$$
f_{\mathrm{ei}}=\left[1-2 a_{\mathrm{e}} \alpha_{0 i}\right]^{-1}
$$

and

$$
f_{i}=\left[1-2 a \alpha_{0 i}\right]^{-1}
$$

where $a=a_{\mathrm{p}}+a_{\mathrm{e}}$. Finally, $\Delta \tilde{\mathbf{m}}_{0}=f_{\mathrm{e} 2} \mathbf{m}_{02}-f_{\mathrm{e} 1} \mathbf{m}_{01}$ and $\Delta \tilde{\alpha}_{0}$ $=f_{\mathrm{e} 2} \alpha_{02}-f_{\mathrm{e} 1} \alpha_{01}$ are the difference dipole moment and polarizability "dressed" with the many-body field of the solvent induced dipoles.
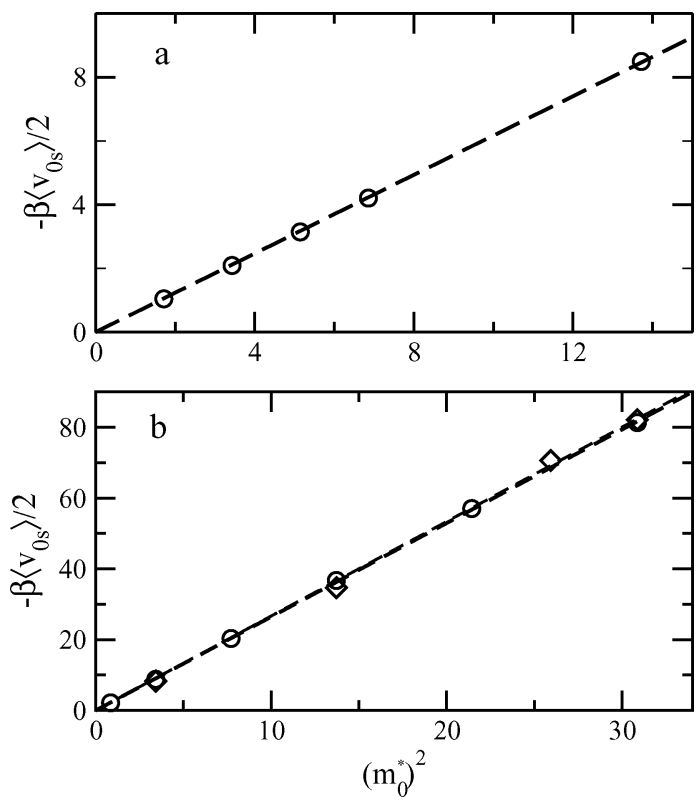

Figure 1. $-\beta\left\langle v_{0 s}\right\rangle / 2$ from eq 27 vs $\left(m_{0}^{*}\right)^{2}=\beta m_{02}{ }^{2} / \sigma_{0}{ }^{3}$ for a solvent with $m=0$ (a) and for a solvent with $\left(m^{*}\right)^{2}=5.0$ (b), $\alpha^{*}=0$ (circles) and 0.06 (diamonds); $\alpha_{0}=0$. Dashed lines are regressions with the slopes 0.617 in (a) and 2.646 (circles) and 2.668 (diamonds) in (b).

Equation 24 for the reorganization energy $\lambda_{\mathrm{p}}$ can be directly tested on computer simulations provided the response coefficients $a_{\mathrm{e}, \mathrm{p}}$ are available. They can be obtained from the average solute-solvent interaction energy as a function of squared solute dipole $m_{0}{ }^{2}$. In the linear response approximation (LRA), onehalf of the interaction energy is equal to the solvation chemical potential. The total response function $a$ and the response to electronic polarization $a_{\mathrm{e}}$ can be obtained as slopes of half the solute-solvent interaction energy

$$
\frac{1}{2}\left\langle v_{0 \mathrm{~s}}\right\rangle=-\frac{1}{2}\left\langle\mathbf{m}_{0} \cdot \mathbf{R}_{0}\right\rangle
$$

vs $m_{0}^{2}$ measured in dipolar-polarizable solvents $(a)$ and in solvents with zero dipole moment $\left(a_{\mathrm{e}}\right)$. In eq $56, \mathbf{R}_{0}$ is the electric field of the solvent at the center of the solute.

A linear dependence of the average solute-solvent interaction energy on $m_{0}^{2}$ holds indeed very accurately both in nonpolar solvents (Figure 1a) and in polar-polarizable solvents (Figure 1b). In this polarity range, the first and second cumulants of the solute-solvent interaction potential measured on solvent configurations in equilibrium with the dipole $m_{0}$ also follow the relation characteristic of the LRA

$$
-\left\langle v_{0 \mathrm{~s}}\right\rangle=\beta\left\langle\left(\delta v_{0 \mathrm{~s}}\right)^{2}\right\rangle
$$

A linear trend of the average solvation energy with squared solute multipole (charge for ion solvation and dipole moment for dipole solvation) is observed in about all calculations ${ }^{34,35}$ and simulations ${ }^{32,36,37}$ performed in dense polar and nondipolar solvents. This linear dependence is often considered to be a sufficient test of the accuracy of the LRA. In terms of energies, the quadratic trend with solute multipole is equivalent to eq $28 .{ }^{32}$ For dynamical solvent response, eq 28 is paralleled by the equality between the time-dependent Stokes shift and equilibrium correlation functions of the solute-solvent interaction energy. ${ }^{38}$ An additional criterion for the linear response can be formulated in terms of the second cumulants of the solute-solvent interaction energy. The second cumulant $\left\langle\left(\delta v_{0 \mathrm{~s}}\right)^{2}\right\rangle_{x}$ calculated on solvent configurations in equilibrium with dipole 
$x$ should be independent of $x$ in the LRA. ${ }^{39}$ Taking $x=0$ and $x=m_{0}$, one gets

$$
\left\langle\left(\delta v_{0 \mathrm{~s}}\right)^{2}\right\rangle_{0}=\left\langle\left(\delta v_{0 \mathrm{~s}}\right)^{2}\right\rangle
$$

where $\langle\ldots\rangle_{0}$ is the statistical ensemble average over the solvent configurations in equilibrium with zero solute dipole (initial configuration for a CS reaction).

It turns out that eq 29 holds in a much narrower range of solvent parameters than does eq $28 .{ }^{33}$ The comparison of second cumulants for CS and CR transitions provides also a more stringent test of nonlinear response in solvation dynamics. ${ }^{40}$ For dipole solvation, the difference between two second cumulants arises from a significant alteration of the local solute-solvent density profile compared to that in the pure solvent each time the reduced solute dipole

$$
m_{0}^{*}=\left(\frac{\beta m_{0}^{2}}{\sigma_{0}{ }^{3}}\right)^{1 / 2}
$$

deviates from the reduced solvent dipole

$$
m^{*}=\left(\frac{\beta m^{2}}{\sigma^{3}}\right)^{1 / 2}
$$

(either upward or downward). Here, $\sigma_{0}$ and $\sigma$ are the solute and solvent diameters, respectively. This picture is also seen in the present simulations. Although a linear trend with $m_{0}^{2}$ and eq 28 both hold accurately for simulations in equilibrium with dipole moment $m_{0}$ (local LRA), the second moment does not show independence of $m_{0}$ (global LRA). As a result, the reorganization energies for $\mathrm{CS}$ transition fall below the $\mathrm{CR}$ reorganization energies by as much as $15 \%$ at $\left(m^{*}\right)^{2}=4.0$. Since the LRA holds locally, we will nevertheless consider the solvent response coefficients as proportionality constants between half of the average interaction energy and $m_{0}{ }^{2}$. However, since the LRA does not hold globally, separate response coefficients will be defined for CS and CR transitions. This procedure is analogous to assuming a time-dependent Gaussian curvature for nonlinear, nonequilibrium solvation dynamics. ${ }^{41}$

For the model system of a point dipole at the center of a spherical solute, an analytical solution for the chemical potential of solvation in polar solvents is available in terms of Padétruncated perturbation series. It gives the response coefficient $a\left(y, r_{0 \mathrm{~s}}, \rho^{*}\right)$ depending on the solute size, solvent density, and the dipolar density $y$ by the following relation: ${ }^{32,42,43}$

$$
a\left(y, r_{0 \mathrm{~s}}, \rho^{*}\right)=R_{\mathrm{eff}}^{-3} y\left[1+\kappa\left(y, r_{0 \mathrm{~s}}\right) y \sigma^{3} I_{0 \mathrm{~s}}^{(3)} / R_{\mathrm{eff}}^{3}\right]^{-1}
$$

where $\rho^{*}=\rho \sigma^{3}, \rho$ is the number density of the solvent, and $r_{0 \mathrm{~s}}$ $=R_{0} / \sigma+0.5$ is the reduced distance of the closest solutesolvent approach, $R_{0}=\sigma_{0} / 2$. The total and electronic response coefficients necessary for the calculation of $\lambda_{\mathrm{p}}$ in eq 24 can be obtained from eqs 10 and 32 as

$$
\begin{gathered}
a_{\mathrm{p}}=a\left(y_{\mathrm{e}}+y_{\mathrm{p}}, R_{0}, \rho^{*}\right)-a\left(y_{\mathrm{e}}, R_{0}, \rho^{*}\right) \\
a_{\mathrm{e}}=a\left(y_{\mathrm{e}}, R_{0}, \rho^{*}\right)
\end{gathered}
$$

Here, $y_{\mathrm{e}, \mathrm{p}}$ refers to the density of induced and permanent dipoles, respectively

$$
\begin{gathered}
y_{\mathrm{e}}=(4 \pi / 3) \rho \alpha^{\prime} \\
y_{\mathrm{p}}=(4 \pi / 9) \beta \rho\left(m^{\prime}\right)^{2}
\end{gathered}
$$

In eq $32, R_{\text {eff }}$ is the effective radius of a dipolar solute. It depends on the solvent density and $r_{0 \mathrm{~s}}$ through the solute-solvent radial distribution function $g_{0 \mathrm{~s}}^{(0)}(r)\left(g_{0 \mathrm{~s}}(r)=0\right.$ at $\left.r<\sigma r_{0 \mathrm{~s}}\right)$

$$
R_{\mathrm{eff}}^{-3}=3 \int_{0}^{\infty} g_{0 \mathrm{~s}}^{(0)}(r)\left(\mathrm{d} r / r^{4}\right)
$$

Further, $I_{0 \mathrm{~s}}^{(3)}$ is a perturbation integral defined on $g_{0 \mathrm{~s}}^{(0)}(r)$ and the solvent-solvent radial distribution function $g_{\mathrm{ss}}^{(0)}(r)$. Both $\sigma^{3} /$ $R_{\mathrm{eff}}{ }^{3}=I_{0 \mathrm{~s}}^{(2)}$ and $I_{0 \mathrm{~s}}^{(3)}$ are tabulated as polynomials of $\rho^{*}=\rho \sigma^{3}$ and $1 / r_{0 \mathrm{~s}}$ (Appendix A). ${ }^{24,32}$ Finally, $\kappa\left(y, r_{0 \mathrm{~s}}\right)$ in eq 32 is an empirical correction introduced for a better agreement of the Padé perturbation formula with computer simulations: ${ }^{32,44}$

$$
\kappa\left(y, r_{0 \mathrm{~s}}\right)=1+\frac{y}{1+y}\left(1-\frac{0.35}{r_{0 \mathrm{~s}}}\right)^{2}
$$

For the calculation of the response coefficients $a$ and $a_{\mathrm{p}}$, one needs an algorithm for the effective condensed-phase dipole moment $m^{\prime}$ and polarizability $\alpha^{\prime}$ entering $y_{\mathrm{p}}$ and $y_{\mathrm{e}}$ in eq 34 . Starting from Wertheim's renormalized perturbation theory $(\mathrm{RPT})^{45}$ several self-consistent schemes to account for multibody induction effects on thermodynamics of polarizable liquids have been proposed. ${ }^{46-48}$ There are slight and normally insignificant differences in these formulations, and we adopt here the procedure given by Joslin et al. ${ }^{47}$ The latter approach allows one to accommodate nonaxial molecular quadrupoles and nonisotropic polarizabilities into one self-consistent formulation. The self-consistent renormalization, following Wertheim, ${ }^{45}$ is normally formulated on two levels. The first level includes the renormalization of the solvent permanent dipole from its gasphase value $m$ to the condensed-phase value $m^{\prime}$ (Wertheim's 1-RPT). This is achieved by iterative solution of the following equation:

$$
m^{\prime}=m\left(1-\alpha C\left(m^{\prime}\right)\right)^{-1}
$$

The scalar $C\left(m^{\prime}\right)$ is obtained by dividing the local electric field acting on a given molecule in the solvent by the effective dipole moment $m^{\prime}$

$$
C\left(m^{\prime}\right)=-\frac{1}{m^{\prime}}\left(\frac{\partial \Delta f}{\partial m^{\prime}}\right)_{\alpha, \rho, \beta}
$$

where $\Delta f$ is the excess free energy per solvent molecule associated with dipole-dipole interactions between them. Equations 37 and 38 assume isotropic polarizability used in the present simulations and can be reformulated for a general case of anisotropic polarizability. ${ }^{45,47}$ On the second level of renormalization (Wertheim's 2-RPT), the solvent molecular polarizability is renormalized from its gas-phase value $\alpha$ to the condensed-phase value $\alpha^{\prime}$. The polarizability $\alpha$ in eq 37 is then replaced with $\alpha^{\prime}$ which is given by the relation

$$
\alpha^{\prime}=\alpha\left(1-\alpha^{\prime} C\left(m^{\prime}\right)\right)^{-1}
$$

For the calculations performed in this paper we used the free energy of dipole-dipole interactions $\Delta f$ in Stell's Padé form. ${ }^{49,50}$ The details of the calculation procedure are outlined in Appendix B.

In Figure 2, the response coefficients calculated according to eq 32 are compared to MC simulations performed in this study and in a previous publication. ${ }^{20}$ Note that the chemical potential of solvation represented by open circles in Figure $2 b$ have been calculated from the Q model incorporating nonlinear solvation effects through the Stokes shift and two second 

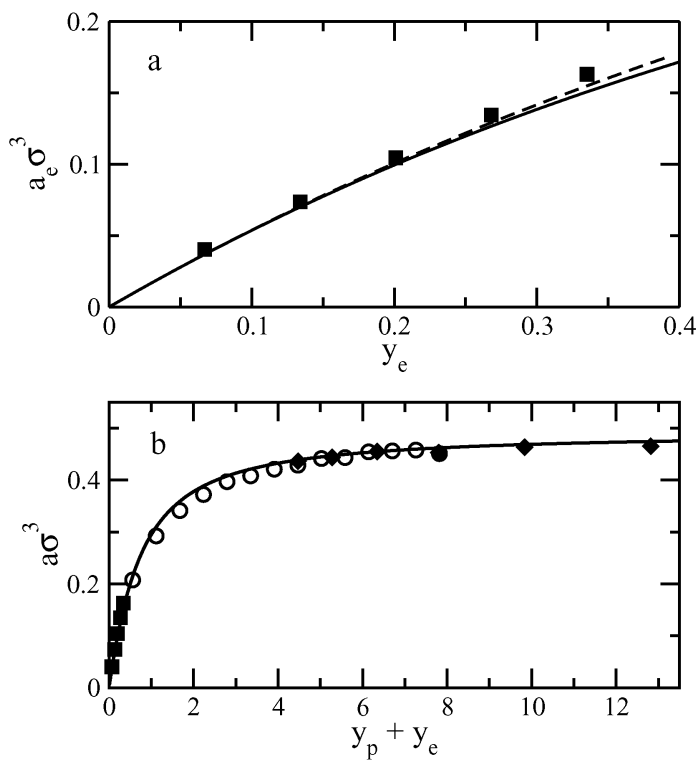

Figure 2. Response coefficients $a_{\mathrm{e}} \sigma^{3}$ (a) and $a \sigma^{3}$ (b) from MC simulations (points) and according to eq 32 (solid lines); $\rho^{*}=0.8, r_{0 \text { s }}$ $=1.4$. In panels $\mathrm{a}$ and $\mathrm{b}$, filled squares indicate the simulation results obtained in polarizable solvents with varying $\alpha$ at $m=0$. In panel $b$, open circles refer to solvents with $\alpha=0$ and varying $m,{ }^{33}$ filled diamonds refer to $\left(m^{*}\right)^{2}=5.0$ and changing $\alpha$, and filled squares refer to $m=0$ and varying $\alpha$. The dashed line in panel $\mathrm{b}$ indicates calculations with $\kappa=1$ in eq 32 .

cumulants of $v_{0 \text { s }}$ corresponding to $\mathrm{CS}$ and $\mathrm{CR}$ transitions. In particular, simulations of dipole solvation in the linear solvation regime do not show saturation of the chemical potential with increasing $y_{\mathrm{p}}$ as would follow from the Onsager form in eq 2. Instead, the chemical potential of linear solvation grows approximately linearly with $y_{\mathrm{p}}$ at $y_{\mathrm{p}} \geq 3$. Only nonlinear solvation, mostly due to partial dewetting of the solute surface in strongly polar solvents, leads to saturation of the solvent response. The Padé form has a quadratic dependence on the solute dipole $\left(\mu \propto m_{0}{ }^{2}\right)$ characteristic of linear response but has saturation incorporated into it to give an accurate account of the MC results. The Padé form should therefore be considered as an empirical approximation that is not capable of distinguishing between linear and nonlinear solvation regimes. The correction factor $\kappa\left(y, r_{0 \mathrm{~s}}\right)$ (eq 36) provides a good global fit of all simulations in nonpolar/polarizable, polar, and polar/polarizable solvents available so far as a function of the dipolar polarity $y_{\mathrm{e}}+y_{\mathrm{p}}$ (solid lines in Figure 2). The data for nonpolar solvents (Figure 2a) are, however, slightly better described by assuming $\kappa=1$ (dashed line in Figure 2) as directly follows from a truncated perturbation expansion for the solvation chemical potential. $^{32}$

The induction reorganization component in eq 23 arises from the modulation of the solute-solvent induction energy gap $\Delta V_{\text {ind }}$ by the solvent density fluctuations. In perturbation models of solvation, this component is given as 24,51

$$
\lambda_{\text {ind }}=\left(f_{\mathrm{e} 2} m_{02}{ }^{2}-f_{\mathrm{e} 1} m_{01}{ }^{2}\right)^{2} \frac{\beta\left(\epsilon_{\infty}-1\right)^{2}}{200 \pi \epsilon_{\infty} \rho^{*} \sigma^{6}} I_{0 \mathrm{~s}}^{(4)}
$$

where the perturbation integral $I_{0 \mathrm{~s}}^{(4)}$ (Appendix A) is

$$
I_{0 \mathrm{~s}}^{(4)}=9 \sigma^{9} \int_{0}^{\infty} \frac{\mathrm{d} r}{r^{10}} g_{0 \mathrm{~s}}^{(0)}(r)
$$

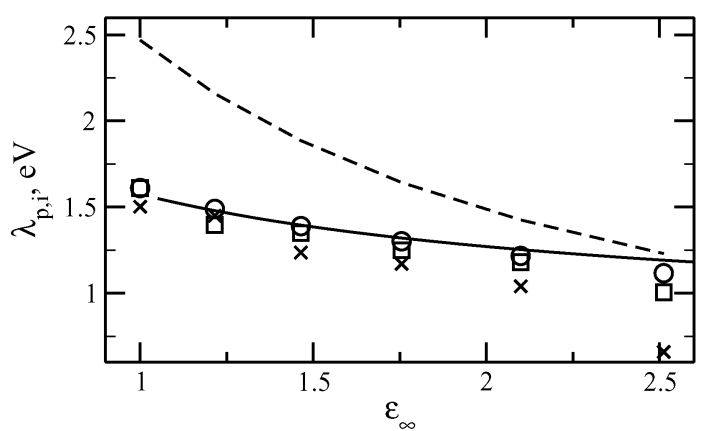

Figure 3. Polar component of the reorganization energy, $\lambda_{\mathrm{p}, i}$, for charge separation (crosses, $i=1$ ) and charge recombination (squares, $i=2$ ) obtained from MC simulations at different values of $\epsilon_{\infty}$. Circles refer to $\lambda_{\mathrm{p}, 2}$ for CR calculated according to eq $47 ;\left(m^{*}\right)^{2}=4.0,\left(m_{0}^{*}\right)^{2}=$ 24.7, $\alpha_{0}=0, \rho^{*}=0.8$. The solid line refers to the analytical theory with the linear response function in the Padé form (eq 32). The dashed line represents the result of dielectric continuum calculation according to eq 48 .

\section{Results}

A. Simulation Procedure. The system investigated in the present paper consists of a spherical solute of the radius $R_{0} / \sigma$ $=0.9$ with a central point dipole $m_{0}$ and isotropic polarizability $\alpha_{0}$ immersed in a solvent of $N=500$ hard sphere (HS) molecules of diameter $\sigma$ with isotropic polarizability $\alpha$, permanent dipole $m$, and the reduced density $\rho \sigma^{3}=\rho^{*}=0.8$. The simulation box is a unit cube centered at the origin with a single solute at its center which does not move and only changes its orientation. The total energy of the mixture includes the dipoledipole interactions between permanent, $\mathbf{m}_{j}^{a}$, and induced, $\mathbf{p}_{j}^{a}$, dipoles and the energy of polarizing the molecules (Drude oscillator model $)^{52-55}$

$$
\begin{aligned}
& U_{\mathrm{tot}}=-\frac{1}{2} \sum_{a, b} \sum_{i, j=1}^{N_{a}, N_{b}}\left(\mathbf{m}_{i}^{a}+\right.\left.\mathbf{p}_{i}^{a}\right) \cdot \mathbf{T}_{i j} \cdot\left(\mathbf{m}_{j}^{b}+\mathbf{p}_{j}^{b}\right)+ \\
& \frac{1}{2} \sum_{a} \sum_{j=1}^{N_{a}} \frac{\left(\mathbf{p}_{i}^{a}\right)^{2}}{2 \alpha_{a}}=-\frac{1}{2} \sum_{a} \sum_{j=1}^{N_{a}} \mathbf{m}_{j}^{a} \cdot \mathbf{E}_{j}
\end{aligned}
$$

where, in our simulations, $a, b=1,2$ and $N_{1}=1, N_{2}=N$. In eq 42

$$
\mathbf{E}_{j}=\sum_{a, k} \mathbf{T}_{j k} \cdot\left(\mathbf{m}_{k}^{a}+\mathbf{p}_{k}^{a}\right)
$$

is the electric field acting on the $j$ th molecule in the liquid. The dipolar tensor $\mathbf{T}_{j k}$ is taken with the reaction field correction for the cutoff of the dipolar interaction potential. ${ }^{29}$ The energy of the $j$ th particle, $-\mathbf{m}_{j}^{a} \cdot \mathbf{E}_{j}$, is updated after each MC move by iterative calculation of the induced dipoles in the simulation box. ${ }^{52}$ The iterations are continued until the relative change in the square of the field $\mathbf{E}_{j}$ was less than $10^{-5}$.

Two sets of simulations, aimed at studying the effects of the solvent and solute polarizability separately, have been carried out. In the first set, we studied $\lambda_{\mathrm{s}}$ at various solvent polarizabilities. Two solute configurations were considered. First, a spherical HS solute with zero dipole $\left(m_{01}=0\right)$ and zero polarizability $\left(\alpha_{01}=0\right)$ was placed in solvents of varying polarizability and constant permanent dipole $\left(\beta m^{2} / \sigma^{3}=\left(\mathrm{m}^{*}\right)^{2}\right.$ $=4.0$ ). These simulations model the reorganization energy of the $\mathrm{CS}$ reaction $\left(0 \rightarrow m_{02}\right.$ transition). The reorganization energy was calculated on MC configurations as the variance of the 
TABLE 1: MC Simulation Results at Varying Solvent Polarizability: $\left(m_{02}^{*}\right)^{2}=24.7, \alpha_{0}=0,\left(m^{*}\right)^{2}=4.0, r_{0 s}=1.4, \rho^{*}=0.8$

\begin{tabular}{|c|c|c|c|c|c|c|c|c|c|c|c|c|c|c|}
\hline$\alpha^{* a}$ & $\epsilon_{\infty}{ }^{b}$ & $y_{p}{ }^{\mathrm{c}}$ & $y_{p}{ }^{d}$ & $-\beta \mu^{e}$ & $-\beta \mu_{e}^{f}$ & $\beta \lambda_{p, 2^{g}}$ & $\beta \lambda_{p, 2^{h}}$ & $\beta \lambda_{p, 1^{i}}$ & $\beta \lambda_{p, 2^{j}}$ & $\beta \lambda_{2}{ }^{k}$ & $\beta \lambda_{1}{ }^{l}$ & $\beta \lambda_{\text {ind }}{ }^{m}$ & $\beta \lambda_{\text {ind }}{ }^{n}$ & $\beta \lambda_{\text {ind }}{ }^{o}$ \\
\hline 0.0 & 1.0 & 4.47 & 4.47 & 62.8 & 0.0 & 62.8 & 62.8 & 58.6 & 63.2 & 62.8 & 58.6 & 0.0 & 0.0 & 0.0 \\
\hline 0.02 & 1.216 & 5.21 & 5.19 & 63.9 & 5.8 & 58.1 & 54.5 & 56.6 & 57.7 & 56.1 & 56.5 & 0.1 & 0.1 & 0.2 \\
\hline 0.04 & 1.464 & 6.21 & 6.24 & 64.0 & 10.6 & 54.9 & 52.6 & 47.6 & 54.4 & 55.2 & 48.2 & 0.3 & 0.7 & 0.7 \\
\hline 0.06 & 1.755 & 7.60 & 7.67 & 65.8 & 15.1 & 50.2 & 48.7 & 44.1 & 51.5 & 51.7 & 45.6 & 0.7 & 1.6 & 1.6 \\
\hline 0.08 & 2.099 & 9.57 & 10.32 & 66.8 & 19.4 & 47.3 & 45.9 & 38.1 & 48.9 & 52.6 & 40.5 & 1.4 & 2.2 & 2.9 \\
\hline 0.10 & 2.512 & 12.48 & 14.96 & 67.0 & 23.5 & 43.5 & 39.2 & 22.6 & 46.5 & 46.0 & 25.7 & 1.6 & 3.1 & 4.5 \\
\hline
\end{tabular}

${ }^{a} \alpha^{*}=\alpha / \sigma^{3} .{ }^{b}$ Calculated from the Clausius-Mossotti equation. ${ }^{c}$ Calculated using the 1-RPT Wertheim theory according to ref $47 .{ }^{d}$ Obtained from simulations by using the average dipole moment $m^{\prime}=\langle|\mathbf{m}+\mathbf{p}|\rangle$ from simulated configurations in the equation $y_{\mathrm{p}}=(4 \pi / 9) \beta \rho\left(m^{\prime}\right)^{2}$. ${ }^{e}$ The total chemical potential of solvation calculated as (LRA) $\mu=\left\langle v_{0 s}\right\rangle / 2 .{ }^{f}$ The chemical potential of solvation due to the induced dipoles of the solvent. Calculated as $\mu_{\mathrm{e}}=\left\langle v_{0 \mathrm{~s}}\right\rangle / 2$ from simulations performed in solvents with $m^{*}=0 .{ }^{g}$ From eq 47. ${ }^{h}$ From simulations as the variance of the solutesolvent coupling in eq 44 evaluated for solvent configurations in equilibrium with a a polar solute (CR configuration of the solute). ${ }^{i}$ From simulations as the variance of the solute-solvent coupling in eq 44 evaluated for solvent configurations in equilibrium with non-polar solute (CS configuration of the solute). ${ }^{j}$ From eqs 24,32 , and $33 .{ }^{k}$ From simulations as the variance of the potential $\Delta V$ (eq 44) evaluated on solvent configurations in equilibrium with the polar solute (CR configuration of the solute). ${ }^{l}$ From simulations as the variance of the potential $\Delta V$ (eq 44 ) evaluated on solvent configurations in equilibrium with the non-polar solute (CS configuration of the solute). ${ }^{m}$ From simulations as the variance of $\Delta V_{\text {ind }}$ in the CR configuration of the solute. ${ }^{n}$ From simulations as the variance of $\Delta V_{\text {ind }}$ in the CS configuration of the solute. ${ }^{\circ}$ Analytical theory, eq 40.

TABLE 2: MC Simulation Results at Varying Solute Polarizability: $\left(m_{02}^{*}\right)^{2}=30.9,\left(m^{*}\right)^{2}=5.0, \alpha / \sigma^{3}=0.06$

\begin{tabular}{|c|c|c|c|c|c|c|c|c|c|c|}
\hline$\alpha_{0}^{* a}$ & $\beta \lambda_{1}{ }^{b}$ & $\beta \lambda_{2}{ }^{c}$ & $\beta \lambda_{p, 1}{ }^{d}$ & $\beta \lambda_{p, 2^{e}}$ & $\beta \lambda_{p, \mathrm{I}^{f}}$ & $\beta \lambda_{p, 2^{g}}$ & $\beta \lambda_{\text {ind }}{ }^{h}$ & $\beta \lambda_{\text {ind }^{i}}{ }^{i}$ & $\beta \hbar \omega_{\mathrm{sl}}{ }^{j}$ & $\gamma^{k}$ \\
\hline 0.00 & 57.0 & 68.8 & 54.7 & 63.6 & 54.7 & 63.2 & 2.1 & 1.5 & 122 & 1.003 \\
\hline 0.02 & 57.5 & 69.6 & 55.1 & 62.8 & 55.2 & 66.5 & 2.1 & 1.4 & 127 & 1.000 \\
\hline 0.04 & 58.0 & 68.5 & 55.6 & 63.1 & 55.7 & 70.0 & 2.1 & 0.8 & 141 & 0.988 \\
\hline 0.06 & 58.5 & 73.3 & 56.0 & 67.5 & 56.1 & 73.9 & 2.2 & 1.0 & 145 & 0.985 \\
\hline 0.08 & 59.0 & 79.8 & 56.5 & 76.3 & 56.6 & 78.0 & 2.2 & 0.9 & 145 & 0.989 \\
\hline 0.10 & 59.5 & 85.4 & 56.9 & 78.9 & 57.1 & 82.3 & 2.2 & 1.0 & 152 & 0.985 \\
\hline 0.12 & 60.0 & 87.6 & 57.4 & 81.5 & 57.6 & 87.2 & 2.3 & 1.8 & 152 & 0.989 \\
\hline
\end{tabular}

${ }^{a} \alpha_{0}{ }^{*}=\alpha_{0} / \sigma^{3} .{ }^{b} \mathrm{CS}$ transition, from simulations as the variance of $\Delta E$ in eq 12. ${ }^{c} \mathrm{CR}$ transition, from simulations as the variance of $\Delta E$. ${ }^{d} \mathrm{CS}$ transition, from simulations as the variance of $\Delta V_{\mathrm{p}}$ (eq 45). ${ }^{e} \mathrm{CR}$ transition, from simulations as the variance of $\Delta V_{\mathrm{p}}$ (eq 45). ${ }^{f} \mathrm{CS}$ transition, calculated from eq 24 with $a_{\mathrm{p}} \sigma^{3}=0.351$ and $a_{\mathrm{e}} \sigma^{3}=0.106 .{ }^{g} \mathrm{CR}$ transition, calculated from eq 24 with $a_{\mathrm{p}} \sigma^{3}=0.304$ and $a_{\mathrm{e}} \sigma^{3}=0.106 .{ }^{h}$ Induction reorganization energy for CS transitions. ${ }^{i}$ Induction reorganization energy for CR transitions. ${ }^{j}$ Stokes shift. ${ }^{k}$ Calculated according to eq 49.

difference interaction potential

$$
\Delta V\left[\mathbf{R}_{\mathrm{p}}, \mathbf{q}\right]=\Delta V_{\mathrm{p}}+\Delta V_{\text {ind }}
$$

where

$$
\Delta V_{\mathrm{p}}=-\mathbf{m}_{02} \cdot \mathbf{f}_{\mathrm{e} 2} \cdot \mathbf{R}_{\mathrm{p}}
$$

and

$$
\Delta V_{\text {ind }}=-\frac{1}{2} \mathbf{m}_{02} \cdot \mathbf{R}_{\infty} \cdot \mathbf{f}_{\mathrm{e} 2} \cdot \mathbf{m}_{02}
$$

Here $m_{02}$ is the solute dipole in the charge-separated state. The same potential difference was used to calculate its variance on the solvent configurations in equilibrium with the solute bearing $\alpha_{02}=0$ and the dipole moment $m_{02}\left(\beta m_{02}{ }^{2} / \sigma_{0}{ }^{3}=24.7\right)$. This procedure gives the reorganization energy of the $\mathrm{CR}$ reaction $\left(m_{02} \rightarrow 0\right.$ transition). To produce sufficient statistics, simulations of the typical length of $6 \times 10^{5}$ cycles were run $(440 \mathrm{~h}$ on the Alpha/ $833 \mathrm{MHz}$ processor) with $2 \times 10^{5}$ production cycles. The simulation results are listed in Table 1 .

The second set of simulations was carried out for a varying solute polarizability at constant solvent polarizability $\left(\alpha / \sigma^{3}=\right.$ $0.06)$ and constant solvent dipole $\left(\left(m^{*}\right)^{2}=5.0\right)$. As above, CS and CR configurations corresponding, respectively, to $m_{01}=0$ and $\beta m_{02}{ }^{2} / \sigma_{0}{ }^{3}=30.9$ were sampled (Table 2). These simulations were $(8-15) \times 10^{5}$ cycles long with $2 \times 10^{5}$ production cycles. The higher the value of the solute polarizability, the longer it took to converge the reorganization energy. The dependence on the solute dipole (Figure 1) gives $a_{\mathrm{e}} \sigma^{3}=0.106$ at $\alpha / \sigma^{3}=$ 0.06 and $m^{*}=0$ and $\left(a_{\mathrm{p}}+a_{\mathrm{e}}\right) \sigma^{3}=0.457$ at $\alpha / \sigma^{3}=0.06$ and $\left(m^{*}\right)^{2}=5.0$.
B. Solvent Polarizability. Figure 2 supports the conclusion of previous simulation studies of solvation ${ }^{14-16}$ and selfconsistent theories of polarizable liquids ${ }^{17}$ that the chemical potential of solvation in nonpolarizable and polarizable dipolar solvents follows the same fundamental law as a function of the polarity parameter $y_{\mathrm{p}}+y_{\mathrm{e}}$. Indeed, results of previous simulations of nonpolarizable solvents ${ }^{33}$ (open circles) and the results of this study for polar/polarizable (filled diamonds) and nonpolar/polarizable (filled squares) solvents all fall on the same theoretical line (eq 32) as a function of $y_{\mathrm{p}}+y_{\mathrm{e}}$ (Figure 2). This result suggests that one fundamental function describes both electronic and nuclear solvation. Therefore, solvation by solely nuclear degrees of freedom can be obtained by subtracting the chemical potential of electronic solvation from the total chemical potential as suggested by eq 10

$$
\lambda_{\mathrm{p}}=-\mu+\mu_{\mathrm{e}}
$$

The direct calculation of $\lambda_{\mathrm{p}}$ for CR coincides very accurately with the calculation according to eq 47 and both results are in good agreement with the analytical theory (eq 32) shown by the solid line in Figure 3. This is the first direct confirmation of the accuracy of eq 47 obtained from computer simulations.

The self-consistent calculations of the effective solvent dipole moment $m^{\prime}$ have been performed here by using the 1-RPT (eq 37) and 2-RPT (eq 39) schemes and compared to direct calculations from MC simulations (third and fourth columns in Table 1, respectively). We found that the 2-RPT renormalization (not shown in Table 1) involving renormalization of the molecular polarizability noticeably overestimates the values of $y_{\mathrm{p}}$ compared to simulations. On the other hand, the 1-RPT scheme (eq 37) provides accurate $y_{\mathrm{p}}$ for all values of $\alpha^{*}$ except the largest one on our list, which is actually very improbable 
for commonly used solvents $\left(\alpha^{*} \simeq 1 / 16\right.$ for most molecular liquids). A similarly good agreement between 1-RPT scheme and simulations of thermodynamics and effective dipole moment of model polarizable liquids has been recently obtained by Boublik and Winkelmann. ${ }^{56}$

The Marcus-Hush formulation applies when the solute polarizability does not change with the electronic transition. The reorganization energy should then be independent of solute's electronic state, i.e., be the same for CS and CR transitions. This prediction holds approximately for the simulation data (Table 1, cf. crosses to squares in Figure 3). A downward deviation of CS reorganization energy from the CR reorganization energy by about $15 \%$ is caused by nonlinear solvation by permanent solvent dipoles. The deviation between CS and CR reorganization energies becomes particularly noticeable at $\alpha^{*}$ $=0.10$. At this polarizability, the dipole-dipole attractions between dipoles $m^{\prime}$ are strong pulling the solvent molecules from the nonpolar solute's surface. The resulting dewetting of solute's surface leads to a lower coordination number of a nonpolar solute compared to a polar solute. The solvating strength of the solvent is reduced resulting in a lower reorganization energy.

Table 1 also presents the splitting of the reorganization energy into the polar and induction components assuming that the modulation of the induction potential and fluctuations of the nuclear reaction field are statistically independent (eq 23). The additive splitting of $\lambda_{\mathrm{s}}$ into $\lambda_{\mathrm{p}}$ and $\lambda_{\text {ind }}$ holds well for CS transitions, and the simulated induction reorganization energies agree remarkably well with eq 40 (cf. two last columns in Table 1). The downward deviation of the last two numbers for CS $\lambda_{\text {ind }}$ can again be traced back to dewetting of solute's surface. For CR transitions, however, $\lambda_{\mathrm{s}}>\lambda_{\mathrm{p}}+\lambda_{\text {ind }}$ at all $\alpha>0$. One can conclude that induction and polar solvation are coupled through the solvent density fluctuations in the presence of a polar solute thus violating the additivity assumption.

The dashed line in Figure 3 refers to the result of dielectric continuum calculation using the $f$ factor from eq 2 . Since the static dielectric constant is very high for the fluid of dipolar spheres lacking the quadrupole moment, ${ }^{17,57}$ the dashed line is plotted assuming $\epsilon_{\mathrm{s}} \rightarrow \infty$ for the continuum $\lambda_{\mathrm{p}}$ :

$$
\lambda_{\mathrm{p}}=\frac{m_{02}{ }^{2}}{{R_{0}}^{3}}\left[0.5-\frac{\epsilon_{\infty}-1}{2 \epsilon_{\infty}+1}\right]
$$

The main qualitative result of this comparison is that the Lippert-Mataga formula for $\lambda_{\mathrm{p}}$ substantially overestimates the slope of its dependence on $\epsilon_{\infty}$.

C. Solute Polarizability. The results of simulations of $\lambda_{\mathrm{s}}$ at constant solvent parameters and varying polarizability of the solute in the final ET state are listed in Table 2. In modeling CS transitions, we assume that the ET system goes from an initial nonpolar, nonpolarizable state with $m_{01}=0, \alpha_{01}=0$ to a polar-polarizable state with $m_{02} / m=6.0$ and varying $\alpha_{02}$. CR refers to the backward transition. As in the case of simulations with changing solvent polarizability, we notice that the splitting of the total reorganization energy $\lambda_{\mathrm{s}}$ into two separate components arising from nuclear polarization and induction interactions (eq 23) is inaccurate for CR transitions. On the contrary, eq 23 holds well for CS transitions (Table 2). This indicates that the nonadditive character of induction and polar contributions to the reorganization energy is caused by the mixing of the fluctuations of induction and polar interaction energies through density fluctuations of the solvent in the presence of a polar solute.

The theoretical prediction for the component of solvent reorganization energy $\lambda_{\mathrm{p}}$ arising from fluctuations of the nuclear

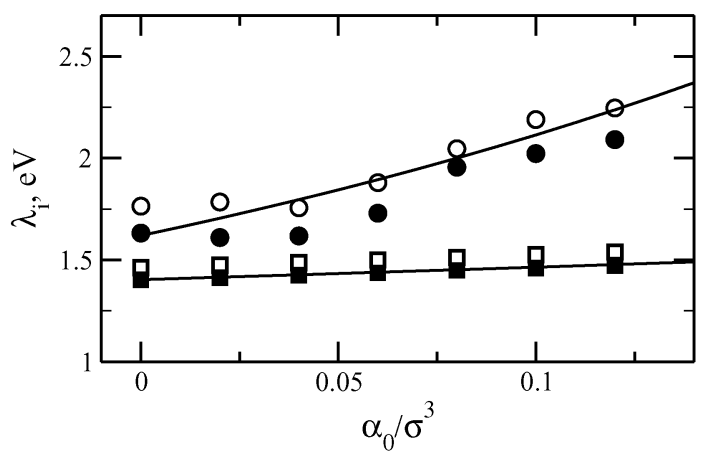

Figure 4. Full reorganization energies $\lambda_{i}$ (open points) and polar reorganization energies $\lambda_{\mathrm{p}, i}$ (filled points) for charge separation (squares, $i=1$ ) and charge recombination (circles, $i=2$ ) obtained from MC simulations as a function of $\alpha_{0} / \sigma^{3}$. The solid lines correspond to $\lambda_{\mathrm{p}, i}$ calculated from eq 24 for CS (lower curve) and CR (upper curve). The response coefficients entering eq 24 are obtained from MC simulations; $\left(m^{*}\right)^{2}=5.0, \alpha^{*}=0.06, \rho^{*}=0.8, r_{0 \mathrm{~s}}=1.4$

reaction field $\mathbf{R}_{\mathrm{p}}$ (eq 24, solid lines in Figure 4) turns out to be quite accurate when tested against simulations. We stress that the response coefficients $a_{\mathrm{e}, \mathrm{p}}$ used to calculate $\lambda_{\mathrm{p}}$ in eq 24 are obtained here directly from simulations. The analysis given in Figure 4 is therefore a direct test of eq 24 independent of solvation models which may be applied to calculate $a_{\mathrm{e}, \mathrm{p}}$. For $\left(m^{*}\right)^{2}=5.0$ and $\alpha^{*}=0.06$ taken for the solvent parameters in this set of simulations the effective dipolar density is $y_{\mathrm{p}}=9.61$. In this polarity range, nonlinear solvation caused by partial dewetting of solute's surface becomes noticeable bringing the CS reorganization energy below the corresponding CR reorganization energy by about $17-20 \%$ even at $\Delta \alpha_{0}=0$. To account for this effect, two different values of the nuclear solvation coefficient, $a_{\mathrm{p}} \sigma^{3}=0.351$ and $a_{\mathrm{p}} \sigma^{3}=0.304$, were adopted for the calculation of $\lambda_{\mathrm{p}}$ for $\mathrm{CR}$ and $\mathrm{CS}$, respectively. The first coefficient is obtained from the dependence of the average solvation energy on the solute dipole (Figure 1). The second coefficient is obtained from the value of solvation energy at $\alpha_{0}$ $=0$. Within simulation uncertainties, there is a good agreement between the analytical prediction (eq 24) and simulated reorganization energies (Figure 4).

The consistency of the Q model leading to eq 24 requires that the reorganization energies for CS and CR transitions are related to the Stokes shift $\hbar \omega_{\mathrm{st}}=\left|\langle\Delta V\rangle_{0}-\langle\Delta V\rangle\right|$ by the parameter $\gamma$ identically equal to unity in the $\mathrm{Q}$ model

$$
\gamma=\frac{\lambda_{\mathrm{s}}^{\mathrm{CS}}}{\lambda_{\mathrm{s}}^{\mathrm{CR}}} \frac{\left(\lambda_{\mathrm{s}}^{\mathrm{CR}}+\hbar \omega_{\mathrm{st}}\right)^{3}}{\left(\lambda_{\mathrm{s}}^{\mathrm{CS}}+\hbar \omega_{\mathrm{st}}\right)^{3}} \equiv 1
$$

The last column in Table 2 lists parameters $\gamma$ obtained from CS and CR reorganization energies and the Stokes shift. As is seen, eq 49 holds very accurately indeed.

\section{Discussion}

Since the understanding of the role of nuclear solvent modes in producing Stokes shifts of optical spectral lines ${ }^{3}$ and the reorganization energy of radiationless transitions ${ }^{1}$ was achieved in $1950 \mathrm{~s}$, the problem of extracting the nuclear component of the solvent response has been involved in all realistic calculations of these properties. The most widely used approach to this problem is to subtract the response of the fast electronic subsystem of the solvent from the total solvent response (eq 10 for the response function and eq 47 for the reorganization energy). Although widely used for calculations of complex molecular systems, the approach itself has never been tested 
on computer simulations and/or experiment. The present simulations provide the first direct test of eqs 10 and 47 convincingly testifying to their accuracy.

The next, more practical, step is to provide an accurate algorithms for calculating the total and electronic responses of the solvent. For this task, continuum solvation models predict a very significant variation of the solvent reorganization energy with the high-frequency dielectric constant $\epsilon_{\infty}$. This prediction is not supported by the current simulations. Instead of a twotimes drop of the reorganization energy predicted by the Lippert-Mataga equation (eq 2) when $\epsilon_{\infty}$ changes from 1.0 to 2.5 , a modest decrease by about $30 \%$ is obtained in MC simulations. The failure of dielectric models is caused by the incorrect calculation of both the total solvation free energy ( $\mu$ in eq 47) and its electronic component ( $\mu_{\mathrm{e}}$ in eq 47). Although $\mu_{\mathrm{e}}$ from simulations shows a linear trend with $\left(\epsilon_{\infty}-1\right) /\left(2 \epsilon_{\infty}+\right.$ 1 ), as predicted by the Onsager model (not shown here), the slope is too high. An accurate formalism to calculate the electronic response is provided by the perturbation expansion (eq 32, Figure 2a). On the other hand, the component $\mu$ from continuum models essentially does not change with $\epsilon_{\infty}$ in highly polar solvents (see eq 48), whereas $\mu$ from simulations increases by about $7 \%$ in the range of $\epsilon_{\infty}$ given in Table 1 . The combination of two errors (in calculating $\mu_{\mathrm{e}}$ and $\mu$ ) leads to a much stronger dependence of $\lambda_{\mathrm{s}}$ on $\epsilon_{\infty}$ in continuum theories than is obtained from both simulations and microscopic analytical models (Figure 3). The analytical Padé approximation for the dipolar solvation energetics (eq 32) has been previously applied to the interpretation of experimental charge-transfer kinetic data. ${ }^{58}$ This is the first extensive test of the model on computer simulations confirming its accuracy in a broad range of solvent polarities.

On the qualitative level, the most interesting finding of the simulations is a noticeable increase of the total solvent response $(\mu)$ with increasing $\epsilon_{\infty}$. This is the result of a linear dependence of the LRA solvation chemical potential on the solvent polarity parameter $y_{\mathrm{p}}+y_{\mathrm{e}}$ for highly polar solvents contrasting with a fast transition to saturation in dielectric theories. ${ }^{33,57}$ In view of the importance of accurate estimates of the dependence of solvation energetics in general and $\lambda_{\mathrm{s}}$ in particular on $\epsilon_{\infty}$, especially for treating solvation at elevated pressure and temperature, experimental verification of the results reported here is required. The dependence of $\lambda_{\mathrm{s}}$ on $\epsilon_{\infty}$ in real chargetransfer systems can be measured by combined alteration of temperature and pressure keeping the total static dielectric constant $\epsilon_{\mathrm{S}}$ invariant (similarly to the isodielectric approach used in photoisomerization kinetics ${ }^{59}$ ) and varying only $\epsilon_{\infty}$.

In contrast to a relatively weak effect of the alteration of solvent polarizability on both $\mathrm{CS}$ and $\mathrm{CR}$ reorganization energies, changing the solute polarizability makes a very significant effect on the CR reorganization energy. The reorganization energy is affected by solute polarizability through the free energy of solute's self-polarization quadratic in the nuclear reaction field of the solvent (eq 22). The chargeseparated state is characterized by a large dipole moment resulting in a large reaction field and high sensitivity of the corresponding reorganization energy to polarizability. A nonpolar state of the solute (CS state in this study) does not produce a substantial reaction field thus leading to a small polarization term and low sensitivity of the reorganization energy to polarizability.

An analytical theory of $\mathrm{ET}^{19}$ provides a route (eq 24) for the calculation of reorganization energy in polarizable dipolar solutes. The simulation data presented here aim at direct testing
TABLE 3: Coefficients of the Density Expansion for the Perturbation Integrals Used in the Calculation of $\lambda_{\mathrm{s}}{ }^{a}$

\begin{tabular}{cccrrr}
\hline integral & coefficients & 0 & 1 & \multicolumn{1}{c}{2} & \multicolumn{1}{c}{3} \\
\hline$I_{0 s}^{(2)}$ & $\mathrm{a}$ & 0.0 & 1.935 & -0.972 & 0.398 \\
& $\mathrm{~b}$ & 0.0 & -1.675 & 2.183 & -0.831 \\
& $\mathrm{c}$ & 0.0 & 0.439 & -1.051 & 0.465 \\
$I_{0 \mathrm{~s}}^{(3)}$ & $\mathrm{a}$ & 1.0 & 0.602 & -0.381 & -0.061 \\
& $\mathrm{~b}$ & $-9 / 16$ & 0.255 & 0.848 & -0.107 \\
& $\mathrm{c}$ & $1 / 32$ & -0.256 & -0.23 & 0.098 \\
$I_{0 \mathrm{~s}}^{(4)}$ & $\mathrm{a}$ & 0.0 & 3.212 & 2.862 & -0.695 \\
& $\mathrm{~b}$ & 0.0 & -2.580 & -4.349 & 3.066 \\
& $\mathrm{c}$ & 0.0 & 0.608 & 1.564 & -1.447
\end{tabular}

${ }^{a}$ The columns labeled $0,1,2$, and 3 stand for the corresponding powers in the polynomial expansions in $\rho^{*}$ as in eq A2.

of this equation with all parameters necessary for the calculations obtained from simulations. A good agreement between the analytical theory and simulation results has been achieved (Figure 4). The present study thus confirms the earlier prediction of analytical models ${ }^{19,25}$ of a significant gap between CS and CR reorganization energies in asymmetric ET systems leading to a pronounced asymmetry of the free energy surfaces of ET. ${ }^{60}$

Acknowledgment. Acknowledgments are made to Research Corporation (RI0748) and to the donors of the Petroleum Research Fund, administered by the ACS (39539-AC6) for support of this research. This is publication 560 from the ASU Photosynthesis center.

\section{Appendix A}

The perturbation integrals in eqs 32 and 40 are calculated by using the HS solute-solvent distribution function obtained from the solution of the Percus-Yevick equation with the contactvalue correction according to ref 61 . The numerical integration is fitted to the polynomials in $1 / r_{0 \mathrm{~s}}$ and $\rho^{*}$ according to the following relations

$$
\begin{gathered}
I_{0 s}^{(2)}\left(r_{0 s}, \rho^{*}\right)=\frac{1}{r_{0 s}{ }^{3}}+\frac{a\left(\rho^{*}\right)}{r_{0 s}{ }^{4}}+\frac{b\left(\rho^{*}\right)}{r_{0 s}{ }^{5}}+\frac{c\left(\rho^{*}\right)}{r_{0 s}{ }^{6}} \\
I_{0 s}^{(3)}\left(r_{0 s}, \rho^{*}\right)=\frac{a\left(\rho^{*}\right)}{r_{0 s}{ }^{3}}+\frac{b\left(\rho^{*}\right)}{r_{0 s}{ }^{4}}+\frac{c\left(\rho^{*}\right)}{r_{0 s}{ }^{6}} \\
I_{0 s}^{(4)}\left(r_{0 s}, \rho^{*}\right)=\frac{1}{r_{0 s}{ }^{9}}+\frac{a\left(\rho^{*}\right)}{r_{0 s}{ }^{10}}+\frac{b\left(\rho^{*}\right)}{r_{0 s}{ }^{11}}+\frac{c\left(\rho^{*}\right)}{r_{0 s}{ }^{12}}
\end{gathered}
$$

where each of the functions $a\left(\rho^{*}\right), b\left(\rho^{*}\right)$, and $c\left(\rho^{*}\right)$ is a thirdorder polynomial in $\rho^{*}$, for example

$$
a\left(\rho^{*}\right)=a_{0}+a_{1} \rho^{*}+a_{2}\left(\rho^{*}\right)^{2}+a_{3}\left(\rho^{*}\right)^{3}
$$

The coefficients $a_{n}, b_{n}$, and $c_{n}$ for each perturbation integral are listed in Table 3.

\section{Appendix B}

The calculation of the effective liquid-state dipole $m^{\prime}$ and polarizability $\alpha^{\prime}$ requires the free energy of interaction between effective dipoles $m^{\prime}$ in eq 38. A convenient approximation for this property is available from the Padé truncation of the perturbation expansion for the free energy per solvent molecule:

$$
\beta \Delta f=f_{2}+f_{3}+\ldots
$$

where each term $f_{n}$ in the expansion is of the $n$th order in the 
dipole-dipole interaction potential. The perturbation expansion is formally diverging at high polarities, but divergence can be eliminated in the Padé approximant ${ }^{50}$

$$
\beta \Delta f_{\mathrm{P}}=f_{2}\left(1-f_{3} / f_{2}\right)^{-1}
$$

In the case of dipole-dipole interaction, the perturbation terms $f_{2}$ and $f_{3}$ are given by the product of powers of the reduced density $\rho^{*}$, reduced dipole moment $m^{*}$, and two perturbation integrals which are defined solely in terms of the reduced density $\rho^{*}:$

$$
\begin{aligned}
& f_{2}=-\left(\rho^{*} m^{*} / 6\right) I_{\mathrm{ss}}^{(2)}\left(\rho^{*}\right) \\
& f_{3}=\left(\rho^{*} m^{*} / 54\right) I_{\mathrm{ss}}^{(3)}\left(\rho^{*}\right)
\end{aligned}
$$

The perturbation integrals $I_{\mathrm{ss}}^{(2,3)}$ are calculated from the solvent-solvent distribution function $g_{\mathrm{ss}}^{(0)}$. According to Larsen et al., ${ }^{49}$ they can be approximated by polynomial expansions in $\rho^{*}$ :

$$
\begin{aligned}
& I_{\mathrm{ss}}^{(2)}\left(\rho^{*}\right)=4.1888+2.8287 \rho^{*}+0.8331 \rho^{*^{2}}+ \\
& 0.0317 \rho^{*^{3}}+0.0858 \rho^{*^{4}}-0.0846 \rho^{* 5} \\
& I_{\mathrm{ss}}^{(3)}\left(\rho^{*}\right)=16.4493+19.8096 \rho^{*}-7.4085 \rho^{*^{2}-} \\
& 1.0792 \rho^{*^{3}}-0.9901 \rho^{*^{4}}-1.0249 \rho^{*^{5}}
\end{aligned}
$$

In self-consistent calculations of the liquid-state dipole moment $m^{\prime}$ according to eq 37 the reduced dipole moment $\left(m^{*}\right)^{2}$ in eq $\mathrm{B} 3$ is replaced with $\beta\left(m^{\prime}\right)^{2} / \sigma^{3}{ }^{47}$

\section{References and Notes}

(1) Marcus, R. A. Rev. Mod. Phys. 1993, 65, 599.

(2) Onsager, L. J. Phys. Chem. 1939, 43, 189.

(3) Mataga, N. Molecular interactions and electronic spectra; Marcel Dekker: New York, 1970.

(4) Hupp, J. T.; Dong, Y.; Blackbourn, R. L.; Lu, H. J. Phys. Chem. 1993, 97, 3278 .

(5) Maroncelli, M.; Fleming, G. R. J. Chem. Phys. 1987, 86, 6221.

(6) Barbara, P. F.; Jarzeba, W. Adv. Photochem. 1990, 15, 1.

(7) Horng, M. L.; Gardecki, J. A.; Papazyan, A.; Maroncelli, M. J. Phys. Chem. 1995, 99, 17311.

(8) Vath, P.; Zimmt, M. B.; Matyushov, D. V.; Voth, G. A. J. Phys. Chem. B 1999, 103, 9130.

(9) Swaddle, T. W. Can. J. Chem. 1996, 74, 631.

(10) Böttcher, C. J. F. Theory of electric polarization; Elsevier: Amsterdam, The Netherlands, 1973.

(11) Tong, G. S. M.; Kurnikov, I. V.; Beratan, D. N. J. Phys. Chem. B 2002, 106, 2381.

(12) Siriwong, K.; Voityuk, A. A.; Newton, M. D.; Rösch, N. J. Phys. Chem. B 2003, 107, 2595.

(13) King, G.; Warshel, A. J. Chem. Phys. 1990, 93, 8682.

(14) Bader, J. S.; Berne, B. J. J. Chem. Phys. 1996, 104, 1293.
(15) Bader, J. S.; Cortis, C. M.; Berne, B. J. J. Chem. Phys. 1997, 106, 2372

(16) Ando, K. J. Chem. Phys. 2001, 115, 5228.

(17) Stell, G.; Patey, G. N.; Høye, J. S. Adv. Chem. Phys. 1981, 18, 183

(18) Liptay, W. Dipole moments and polarizabilities of molecules in excited electronic states. In Excited States; Lim, E. C., Ed.; Academic Press: New York, 1974; Vol. 1.

(19) Matyushov, D. V.; Voth, G. A. J. Phys. Chem. A 1999, 103, 10981. 8516 .

(21) Matyushov, D. V.; Ladanyi, B. M. J. Chem. Phys. 1998, 108, 6362. (22) Gehlen, J. N.; Chandler, D.; Kim, H. J.; Hynes, J. T. J. Phys. Chem. 1992, 96, 1748 .

(23) Marcus, R. A. J. Phys. Chem. 1989, 93, 3078.

(24) Matyushov, D. V.; Schmid, R. J. Chem. Phys. 1995, 103, 2034.

(25) Matyushov, D. V.; Voth, G. A. J. Chem. Phys. 1999, 113, 5413.

(26) Pratt, L. R. Mol. Phys. 1980, 40, 347.

(27) Thompson, M. J.; Schweizer, K. S.; Chandler, D. J. Chem. Phys. 1982, 76, 1128 .

(28) Cao, J.; Berne, B. J. J. Chem. Phys. 1993, 99, 2902.

(29) Allen, M. P.; Tildesley, D. J. Computer simulation of liquids; Clarendon Press: Oxford, U.K., 1996.

(30) Rick, S. W.; Stuart, S. J. Potentials and algorithms for incorporating polarizability in computer simulations. Reviews in Computational Chemistry; Wiley-VCH: New York, 2002; Vol. 18.

(31) Hemmer, P. C.; Holden, H.; Ratkje, S. K., Eds.; The collected works of Lars Onsager; World Scientific: Singapore, 1996.

(32) Matyushov, D. V.; Ladanyi, B. M. J. Chem. Phys. 1999, 110, 994

(33) Milischuk, A.; Matyushov, D. V. J. Phys. Chem. A 2002, 106, 2146.

(34) Fonseca, T.; Ladanyi, B. M.; Hynes, J. T. J. Phys. Chem. 1992, 96, 4085 .

(35) Chong, S.-H.; Miura, S.; Basu, G.; Hirata, F. J. Phys. Chem. 1995, 99, 10526.

(36) Maroncelli, M. J. Chem. Phys. 1991, 94, 2084.

(37) Ichiye, T. J. Chem. Phys. 1996, 104, 7561.

(38) Bagchi, B.; Chandra, A. Adv. Chem. Phys. 1991, 80, 1.

(39) Carter, E. A.; Hynes, J. T. J. Chem. Phys. 1991, 94, 5961.

(40) Matyushov, D. V. J. Chem. Phys. 2001, 115, 8933.

(41) Geissler, P. L.; Chandler, D. J. Chem. Phys. 2000, 113, 9759.

(42) Matyushov, D. V.; Schmid, R. J. Chem. Phys. 1996, 105, 4729.

(43) Matyushov, D. V.; Voth, G. A. J. Chem. Phys. 1999, 111, 3630.

(44) The empirical parameter of the original formulation in ref $32 \kappa_{\mathrm{p}}=$ $1+\left(1-0.5 / r_{0 \mathrm{~s}}\right)^{2}$ is slightly modified here to account for an extended range of simulation data from ref 33 and from the present simulations.

(45) Wertheim, M. S. Mol. Phys. 1979, 37, 83.

(46) Carnie, S. L.; Patey, G. N. Mol. Phys. 1982, 47, 1129.

(47) Joslin, C. G.; Gray, C. G.; Gubbins, K. E. Mol. Phys. 1985, 54, 117.

(48) Høye, J. S.; Lomba, E. Mol. Phys. 1994, 81, 385.

(49) Larsen, B.; Rasaiah, J. C.; Stell, G. Mol. Phys. 1977, 33, 987.

(50) Stell, G. Fluids with long-range forces; Toward a simple analytic theory. In Statistical Mechanics. Part A: Equilibrium Techniques; Berne, B. J., Ed.; Plenum: New York, 1977.

(51) Matyushov, D. V.; Schmid, R. Mol. Phys. 1995, 84, 533.

(52) Vesely, F. J. J. Comput. Phys. 1976, 24, 361.

(53) Patey, G. N.; Torrie, G. M.; Valleau, J. P. J. Chem. Phys. 1979, $71,96$.

(54) Pollock, E. L.; Alder, B. J.; Patey, G. N. Physica A 1981, 108, 14.

(55) Kriebel, C.; Winkelmann, J. Mol. Phys. 1996, 88, 559.

(56) Boublik, T.; Winkelmann, J. Mol. Phys. 1999, 96, 435.

(57) Milischuk, A.; Matyushov, D. V. J. Chem. Phys. 2003, 118, 1859

(58) Zimmt, M. B.; Waldeck, D. H. J. Phys. Chem. A 2003, 107, 3580

(59) Anderton, R. M.; Kauffman, J. F. J. Phys. Chem. 1994, 98, 12125.

(60) Small, D. W.; Matyushov, D. V.; Voth, G. A. J. Am. Chem. Soc. $\mathbf{2 0 0 3}, 125,7470$

(61) Lee, L. L.; Levesque, D. Mol. Phys. 1973, 26, 1351. 